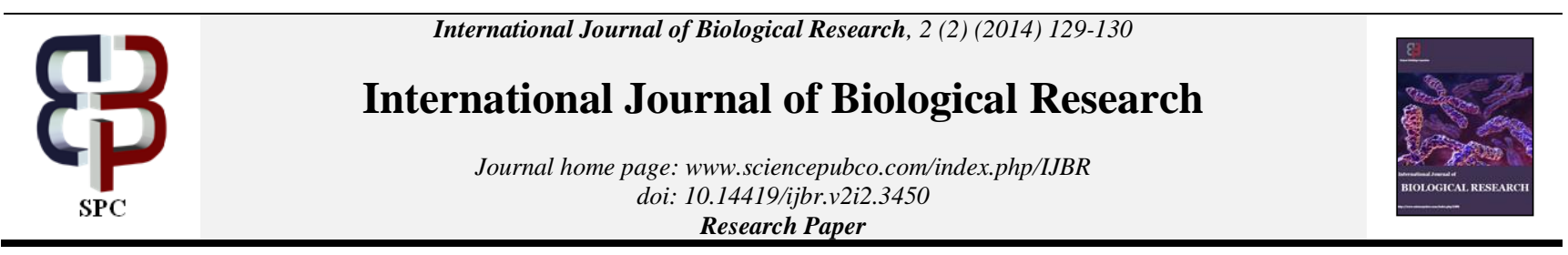

\title{
Comparative screening of enzyme producing endophytic actinomycetes from fresh and fallen leaves of Emblica officinalis in Western Ghats
}

\author{
Mrinalini.J.Singh ${ }^{1}$, Dr.S.Padmavathy ${ }^{2}$ \\ ${ }^{1}$ Research Scholar, Department of Botany, Nirmala College for Women, Coimbatore 641 018, Tamil Nadu, India \\ ${ }^{2}$ Associate Professor of Botany, Nirmala College for Women, Coimbatore 641 018, India \\ *Corresponding author E-mail: mrinalini.singh50@gmail.com
}

\begin{abstract}
Objective: To assess the comparative amylase, lipase and cellulase production abilities of endophytic actinomycetes isolated from both fresh and fallen leaves of Emblica officinalis in Western Ghats.

Methods: Endophytic actinomycetes were isolated using Starch casein agar media. The screening of these isolates for extracellular enzymatic production was carried out using carbohydrate degradation test.

Results: Among the endophytic actinomycetes isolated from fresh leaves, 75\% exhibited amylase activity, $41.66 \%$ exhibited lipase activity and 50\% exhibited cellulase activity whereas in fallen leaves, $80 \%$ exhibited amylase activity, 60\% exhibited lipase activity and $60 \%$ exhibited cellulase activity.

Conclusion: Hence, endophytic actinomycetes isolated from the fallen leaves exhibited remarkable lipase, cellulase and amylase producing capacity which mediates as biocatalysts in bioremediation and biofuel production, as compared to fresh leaves.
\end{abstract}

Keywords: Amylase, Lipase, Cellulase, Endophytic Actinomycetes, Emblica officinalis, Western Ghats.

\section{Introduction}

Accumulation of pollutants seems to be a crucial factor in this global world due to non-availability of cost effective waste treatment. Physical chemical waste treatment proves to be too costly. Even, the necessity of biofuel production increases due to global shortage of fossil fuels and tremendous increase in crude oil price. Enzyme mediated catalysis has got more advantages since it does not create much environmental concern when compared to chemical mediated catalysis. Several microbes are also involved in the hydrolysis of organic pollutants (Emimol A et al., 2012) mediated by extra cellular hydrolytic enzymes for speeding up the biological waste treatment process. These enzymes are also used as biocatalysts in biofuel production apart from waste treatment process. Hence, production of biofuels mediated by lipase, amylase and cellulase enzyme producing microbes are strongly recommended as they prove to be cost effective.

Keeping this cost effective multitude green based applications in mind, an attempt has been made to screen the microbes that can easily breakdown the complex material, mediated by their extracellular hydrolytic enzymes. Microbial screening was specifically focused on actinomycetes since lesser work has been carried out in detail compared to bacteria and fungi. The basic principle of effective remediation technology is nothing but reusing the waste to produce the utilizable product. Even the fallen leaves are treated as wastes. Therefore, both the fresh leaves and fallen leaves were treated as source in order to isolate enzyme producing abilities of endophytic actinomycetes.
Hence, a comparative study has been carried out between the enzyme producing abilities of endophytic actinomycetes from fresh and fallen leaves of common medicinal tree Emblica officinalis in Western Ghats, India.

\section{Materials and methods}

\subsection{Sample collection}

Healthy and fallen leaf samples of common medicinal plant Emblica officinalis with high medicinal value were gathered from the Garden of Nirmala College for Women, Coimbatore (11.0183 ${ }^{\circ}$ $\mathrm{N}, 76.9725^{\circ} \mathrm{E}$ ) which comes under the region of Western Ghats of Southern India during the period of December 2011 to January 2012. The collected plant materials were taken to the laboratory, preserved at $4{ }^{\circ} \mathrm{C}$ in sealed plastic bags and subjected to isolation work within 96 hours.

\subsection{Isolation of endophytic actinomycetes}

Healthy leaf samples were cut into small pieces $(2 * 2 \mathrm{~cm})$ and washed by running tap water for 1-2 minutes to remove the soil particles completely. The resultant were subjected to a five-step surface sterilization procedure as per the method of Qin et al 2009: a 4 to 10-minutes wash in 5\% sodium hypochlorite, followed by a 10 -minutes wash in $2.5 \%$ sodium sulphite, a 5 -minutes wash in $75 \%$ ethanol, a wash in sterile water, and a final rinse in $10 \%$ sodium bicarbonate for 10 minutes to disrupt the plant tissues and inhibit the fungal growth. At this point, the final washed solution was spread onto Yeast extract Malt extract agar containing 
nalidixic acid $(50 \mathrm{mg} / \mathrm{lit})$ and nystatin $(100 \mathrm{mg} / \mathrm{lit})$ and observed for microbial growth to validate the surface sterilized protocol. After the sterility check, the surface sterilized tissues were subjected to continuous drying at $100^{\circ} \mathrm{C}$ for 15 minutes. Surfacetreated tissues were then inoculated on starch casein agar at supplemented with nalidixic acid (50 mg/lit) and nystatin (100 $\mathrm{mg} / \mathrm{lit})$. The media was then incubated at room temperature $(28 \pm$ $2^{\circ} \mathrm{C}$ ) for 2 to 8 weeks in order to isolate endophytes. The pure cultures were identified by their morphology and colony characteristics. The organisms were maintained on Starch casein agar slant and stored at $4^{\circ} \mathrm{C}$.

\subsection{Screening of isolates for amylase production}

Starch agar plate was inoculated with the organism to be tested and incubated at optimum temperature for at least 48 hours. Then, the plate was flooded with iodine and the results were observed. A clear zone indicates presence of hydrolysis while no clearing indicates absence of hydrolysis.

\subsection{Screening of isolates for lipase production}

Pure cultures of test isolates were aseptically streaked in Tween 80 agar. After incubation for 48 hours, if there arises a turbid zone around the colonies, it proves that esterase will hydrolyze the tween and subsequent precipitation with the calcium chloride will lead to turbid zone formation.

\subsection{Screening of isolates for cellulase production}

The isolates were grown in carboxymethylcellulose (CMC) agar flooded with Congo red at $37^{\circ} \mathrm{C}$ for 2-3 days. A clear zone around the colonies of the isolate is an indication that the isolate is metabolizing the substrate and thus, there is enzyme production/activity (Nwagu K.E. et al., 2012).

\subsection{Identification}

The actinobacterial isolates were presumptively identified by means of macroscopic and microscopic characterization and then compared using actinomycetes identification key (Shirling et al., 1966; Williams et al., 1989).

\section{Results}

Endophytic actinomycetes were isolated from surface sterilized fresh and fallen leaves of Emblica officinalis. A total of 12 and 10 endophytic actinomycetes from fresh and fallen leaves respectively, were recovered on Starch casein agar. Comparative enzyme producing capacity of all these isolates were studied by performing amylase test (Starch hydrolysis), lipase test (Tween 80 hydrolysis) and cellulase test.

Among the endophytic actinomycetes isolated from fresh leaves, $75 \%$ exhibited amylase activity, $41.66 \%$ exhibited lipase activity and $50 \%$ exhibited cellulase activity whereas in fallen leaves, $80 \%$ exhibited amylase activity, 60\% exhibited lipase activity and $60 \%$ exhibited cellulase activity by occurrence of hydrolytic zone around the colonies after 3 days. Based on actinomycetes identification key (Shirling et al., 1966; Williams et al., 1989), the isolated microbes were assigned to the genus Streptomyces.

Table 1: Enzymatic Activities of Endophytic Actinomycetes from Fresh Leaves of Emblica officinalis

\begin{tabular}{llll}
\hline No. of actinomycetes isolates & Amylase & Lipase & Cellulase \\
\hline A1 & + & - & + \\
A2 & + & - & + \\
A3 & - & - & - \\
A4 & - & + & - \\
A5 & + & - & + \\
A6 & + & + & + \\
A7 & - & - & -
\end{tabular}

\begin{tabular}{llll} 
A8 & + & - & - \\
A9 & + & + & + \\
A10 & + & + & - \\
A11 & + & - & + \\
A12 & + & + & - \\
\hline
\end{tabular}

Table 2: Enzymatic Activities of Endophytic Actinomycetes from Fallen Leaves of Emblica officinalis

\begin{tabular}{llll}
\hline No. of actinomycetes isolates & Amylase & Lipase & Cellulase \\
\hline A1 & + & + & + \\
A2 & + & + & - \\
A3 & + & - & + \\
A4 & + & + & + \\
A5 & - & - & - \\
A6 & + & + & - \\
A7 & + & + & + \\
A8 & + & + & + \\
A9 & + & - & + \\
A10 & - & - & - \\
\hline
\end{tabular}

\section{Discussion}

Many endophytic actinomycetes isolated from the fallen leaves of Emblica officinalis in Western Ghats exhibited remarkable lipase, cellulase and amylase producing capacity which mediates as biocatalysts in bioremediation and biofuel production respectively, as compared to fresh leaves. The methodology can be further refined by considering the factors (incubation time, $\mathrm{pH}$, aeration, agitation, and temperature and energy source) for the maximized enzyme production to enhance the biodegradation of organic wastes and biofuel production.

\section{Conclusion}

Hence, it can be concluded that endophytic actinomycetes from the plant wastes like fallen leaves of medicinal plants especially rare and endangered plants could be the promising resource of potential biocatalysts for eco-friendly, biodegradable, non-toxic biofuel production which acts as an alternative source of renewable energy and they should be investigated for a safe green future.

\section{Acknowledgments}

We are thankful to authorities of Nirmala College for Women, Coimbatore, and Tamil Nadu for providing necessary facilities. The first author is sincerely grateful to University Grants Commission, Government of India, for providing the award of Junior Research Fellowship in Science to pursue the research work.

\section{References}

[1] Emimol A, Ganga.G, Parvathy. R, Radhika.G, Nair G.M. 2012 Screening of microbes producing extracellular hydrolytic enzyme from corporation waste dumping site and house hold waste for the enhancement of bioremediation methods. IOSR Journal of Pharmacy and Biological Sciences 4(1):54-60. http://dx.doi.org/10.9790/30080415456.

[2] Qin, S., Li, J., Chen, H.-H., Zhao, G.-Z., Zhu, W.-Y., Jiang, C.-L., et al. 2009. Isolation, diversity, and antimicrobial activity of rare actinobacteria from medicinal plants of tropical rain forests in Xishuangbanna China, Appl. Environ. Microbiol. 75: 6176-6186. http://dx.doi.org/10.1128/AEM.01034-09.

[3] Nwagu K.E., Ominyi M. C., Nwoba G.E. 2012. Isolation, screening and measurement of amylase and cellulase activities of some microorganisms. Continental J. Biological Sciences 5 (1): 37 - 41.

[4] Shirling, E.B. and Gottlieb,D., 1966. Methods for characterization of Streptomyces species. Int J Syst Bacteriol. 16:313-340. http://dx.doi.org/10.1099/00207713-16-3-313.

[5] Williams,S.T,Sharpe,M.E.and Holt,J.G.,1989. Bergey's Manual of Systematic Bacteriology. Vol 4. Williams and Wilkins, Baltimore. 